\title{
Aislamiento presuntivo y caracterización de Cryptococcus neo- formans y Cryptococcus gattii desde árboles en la región de O’Higgins y Maule, Chile
}

(Presumptive isolation and characterization of Cryptococcus neoformans and Cryptococcus gattii from trees in the region of O'Higgins and Maule, Chile)

Viviana Toro Zúñiga ${ }^{1 *}$, Pedro Brevis Azocar' ${ }^{1}$ ${ }^{1}$ Departamento de Microbiología, Universidad de Talca, Chile. Avenida Lircay s/n Talca. Teléfono: 71-2200452. *Autor para correspondencia: toro.viviana@gmail.com

RECIBIDO:7 de Septiembre de 2015 APROBADO:13 de Octubre de 2015

LOS AUTORES DECLARAN NO TENER CONFLICTO DE INTERESES

Palabras claves: Cryptococcus neoformans, Cryptococcus gattii.

Key words: Cryptococcus neoformans, Cryptococcus gattii.

\section{RESUMEN}

Introducción: La criptocococis es una micosis sistémica causada por $C$. neoformans y $C$. gattii, es frecuente y oportunista en inmunocomprometidos y patógeno primario en personas inmunocompetentes. C. neoformans tiene una distribución mundial y se ha aislado desde las excretas de palomas. C. gattii se considera restringida a regiones con clima tropical, subtropical, y templadas, se encuentra asociada frecuentemente a detritos de especies de Eucalyptus sp. La virulencia de estas levaduras le permite desarrollar patogénesis en mamíferos y supervivencia en el ambiente.

Objetivo: Identificar y determinar la actividad de proteinasas y fosfolipasas, de C. neoformans y $C$. gattii aisladas desde las oquedades de árboles en lugares con alta afluencia de público.

Materiales y Métodos: Se tomaron 200 muestras de hisopado desde distintas especies de árboles desde sectores de la región de O'Higgins y el Maule. Se siembran en ASG, se aíslan y mantienen en ASD.
Identificación con tinta china, Urea de Christensen, crecimiento a $37^{\circ} \mathrm{C}$, asimilación y fermentación de azucares, y siembra en medio CGB. Se mide índice de actividad enzimática Prz de proteinasas y fofolipasas.

Resultados y Conclusiones: Se obtuvieron 109 cepas de C. neoformans aisladas desde las oquedades de diferentes especies arbóreas y 3 cepas presuntivas de C. gattii desde Eucalyptus sp. y Prunus cerasifera artropurpurea. El 88,1\% de las cepas $C$. neoformans y $100 \%$ de C. gattii, presentaron alta actividad proteolítica, El $49,5 \%$ de las cepas de C. neoformans y $33,3 \%$ de C. gattii mostraron alta actividad de fosfolipasas.

\section{ABSTRACT}

Introduction: Criptocococis is a systemic mycosis caused by $C$. neoformans and C. gattii, frequent and opportunistic in immunocompromised and primary pathogen in immunocompetent persons. C. neoformans has a worldwide distribution and 
Aislamiento presuntivo y caracterización de Cryptoccoccus neoformans y Criptococcus gatti - V. Toro et al

has been isolated from the excreta of pigeons. $C$. gattii is considered restricted to regions with tropical, subtropical, and temperate, is often associated with species of Eucalyptus sp. The virulence of these yeasts develop pathogenesis allows survival in mammals and the environment.

Objective: To identify and determine the activity of proteinases and phospholipases of $C$. neoformans and $C$. gattii isolated from the hollows of trees in places with high turnout.

Materials and Methods: 200 swab samples were taken from different species of trees from areas of the region of O'Higgins and Maule. Planted in ASG, they are isolated and kept in ASD. Identification with ink, Urea Christensen, growth at $37^{\circ} \mathrm{C}$, assimilation and fermentation of sugars, and planting medium CGB. Prz index proteinase enzyme activity is measured and phospholipases.

Results and Conclusions: We manage to get 109 strains of $C$. neoformans isolated from the hollows of different tree species and 3 presumptive strains of C. gattii from Eucalyptus sp. and Prunus cerasifera artropurpurea. $88.1 \%$ of the strains C. neoformans and C. gattii 100\%, they showed high proteolytic activity, $49.5 \%$ of the strains of C. neoformans and C. gattii 33.3\% showed high activity phospholipases.

\section{INTRODUCCIÓN}

El género Cryptococcus comprende levaduras encapsuladas saprofitas del ambiente (26), aisladas por primera vez por Sanfelice (1894) desde el jugo de durazno (9), capaces de producir lesiones en animales de experimentación (31). En 1895, Busse y Buschke lo aíslan en humanos, desde lesiones cutáneas y óseas $(9,8)$.

Se conocen un total de 37 especies del género Cryptococcus (26), de las cuales, solo el Complejo Cryptococcus neoformans ha sido considerado como patógeno, causante de criptococcosis en pacientes inmunodeprimidos e inmunocompetentes (14). La infección es por la inhalación de propágu- los ambientales capaces de ingresar a los pulmones y de allí diseminarse sistémicamente por vía hematógena, mostrando un fuerte neurotropismo. (13) De acuerdo a propiedades básicas inmunológicas de los polisacáridos capsulares se reconocen los serotipos A, B, C y D $(7,4)$. Los serotipos A, D y AD corresponden a $C$. neoformans var. neoformans y los serotipos B y C a C. neoformans var. gattii $(12,5)$. Los análisis moleculares, como patrones de huellas dactilares de $\mathrm{ADN}$, reconocen al serotipo D como C.neoformans var. neoformans y al serotipo A como C.neoformans var. grubii (7), y un hibrido $C$. neoformans AD (27). Por su parte C.neoformans var. gattii, desde 2002 se ha considerado una especie separada de $C$. neoformans, denominándose solo C. gattii (24).

Las infecciones causadas por $C$. neoformans se conocen desde 1900, los cuales aumentaron desde la aparición del SIDA en África entre los años 1947-1968 en la cuenca del rio Congo. (34). El primer caso de criptococosis en humanos se le atribuye directamente al medio ambiente contaminado con excrementos de palomas (25). C. neoformans var. grubii tiene distribución mundial, asociado a excrementos de palomas y detritus de árboles (33). En cambio, C. gattii presentaba una distribución restringida a países tropicales y subtropicales asociado a especies de Eucalyptus sp. (13). La distribución ecológica de $C$. gattii se ha ampliado a regiones templadas, quedando demostrado en la epidemia de criptocococis que asotó a la isla de Vancouver en la Británica (BC) en 1999 (11). Cobrando importancia por su capacidad de infectar tanto a individuos inmunocomprometidos como inmunocompetentes (34). C. gattii se ha aislado desde eucaliptus en California, Colombia, Argentina, Brasil, India y Egipto, y desde otras especies arbóreas (22), asociado a huecos deteriorados, en los troncos y/o establecimientos en más de 50 especies de angiospermas y gimnospermas. El creciente volumen de datos de muestreo, es compatible con la idea de que $C$. gattii es de larga data entre la flora fúngica de árboles nativos en muchas 
Aislamiento presuntivo y caracterización de Cryptoccoccus neoformans y Criptococcus gatti - V. Toro et al

partes del mundo (34).

El origen de la virulencia en los hongos ambientales resulta ser un enigma. $C$. neoformans y $C$. gattii poseen factores de virulencia para la patogénesis de mamíferos y para su supervivencia en el ambiente. Estos factores podrían originarse a partir de las presiones de selección impuestas por los depredadores ambientales (35). Los factores de virulencia más estudiados son: crecimiento a $37^{\circ} \mathrm{C}$ $(6,35)$, la producción del polisacárido capsular (6), enzimas extracelulares como proteinasas (32), ureasas $(2,37,38)$, fosfolipasas $(7,29,37)$ y fenoloxidasa $(4,39)$.

El objetivo de esta investigación es identificar y determinar la actividad de proteinasas y fosfolipasas, de C. neoformans y C. gattii, aisladas desde las oquedades de árboles, con alta afluencia de público, en sectores de la región de O’Higgins y el Maule, Chile.

\section{MATERIALES Y MÉTODOS}

Área de estudio

La toma de muestra se realizó entre marzo y mayo del 2012, en plazas y sectores urbanos, con gran afluencia de público, de las localidades de Coya (34¹0'50" S/ 7044’00” O), Machalí (341'00" S/7040'00" O), Rancagua (3410'00" $\left.\mathrm{S} / 70^{\circ} 45^{\prime} 00^{\prime} \mathrm{O}\right)$, Graneros (3404'00” S/7044'00" O) y San Francisco de Mostazal (3359'00" $\mathrm{S} / 70^{\circ} 42^{\prime} 00^{\prime}$ ' O), pertenecientes a la región del Libertador Bernardo O'Higgins, y Talca de la región del Maule (35²6’00” S/7140’00” O), desde sectores como la Estación de Ferrocarriles (EFE), plaza de Armas, Alameda y Universidad de Talca.

Obtención de las muestras

Se tomaron 200 muestras de hisopado estéril en solución salina con cloranfenicol (0,2 g.L-1), desde distintas especies de árboles que presentaban oquedades o irregularidades en su corteza, protegidas de la luz y mantenidas a $4{ }^{\circ} \mathrm{C}$ por un tiempo máximo de una semana, $\mathrm{y}$ transportadas al
Instituto de Microbiología de la Facultad de Ciencias de la Salud de la Universidad de Talca.

Procesamiento de las muestras

Cada muestra hisopada fue inoculada en placas con medio de cultivo agar Semillas de Girasol (ASG) (Helianthus annus), e incubadas por 5 días a $28^{\circ} \mathrm{C}$, observando la aparición de colonias levaduriformes mucosas de color café presuntivas para Cryptococcus sp., las cuales fueron aisladas y resembradas en agar Sabouraud dextrosa (ASD) para estudios posteriores. Se usaron las cepas C. neoformans ATCC 32045 y C. gattii ATCC MYA-4560 como controles positivos.

De las colonias presuntivas se realizaron preparaciones con tinta china para observar la morfología microscópica de la presencia de la cápsula característica del género Cryptococcus. Se realizó la prueba bioquímica de producción de ureasas en medio inclinado de Urea de Christensen. La diferenciación entre C. neoformans de $C$. gattii se realizó con el medio agar canavanina-glicina-azul de bromotimol (CGB) propuesta en 1882 por Kwon-Chung et. al (23).

La medición de proteinasas extracelulares se determinó con la técnica modificada utilizada por Ruma-Haynes et. al (32). Las cepas aisladas fueron sembradas en agar ASD por 24 horas a $30^{\circ} \mathrm{C}$. A partir de estas colonias se prepararon inóculos de $5 \mathrm{~mL}$ de agua destilada estéril con un absorbancia de $0,075 \mathrm{~nm}(\lambda=590 \mathrm{~nm})$. La actividad proteolítica extracelular de las cepas se evaluó preparando una solución de levaduras con una concentración al 0,5 McFarland (1,5 x 108 UFC), inoculando $10 \mu \mathrm{L}$ en el medio de cultivo, e incubadas a $30^{\circ} \mathrm{C}$ por 14 días.

La actividad enzimática extracelular de las fosfolipasas se determinó por el método modificado descrito por Price et. al (30). Se preparan inóculos de las cepas aisladas de $10 \mu \mathrm{L}$, sobre placas ASD suplementado con yema de huevo, incubadas a $30^{\circ} \mathrm{C}$ por 10 días (15).

Las cepas con actividad de proteinasas y fosfo- 
lipasas formaron zonas clarificadas en el medio de cultivo, se midieron los diámetros de las colonias calculando el coeficiente de actividad enzimática (Prz) descrito por Price et al. 1982 (30), que se obtiene al dividir el diámetro de crecimiento de la colonia entre el diámetro de la zona de producción enzimática, medición semicuantitativa de la actividad enzimática (29), toma valores de 0 a 1 , las más cercanas a 0 , serán las cepas que presentaron una mayor actividad enzimática y las cepas cercanas a 1 presentaran un bajo nivel y las que dan un valor igual a 1, no presentaron actividad de esta enzima.

\section{RESULTADOS}

De un total de 200 muestras obtenidas a partir de la madera en descomposición presente en oquedades de troncos de diferentes géneros y especies de árboles, de sectores de la sexta y séptima región, se aíslan 433 colonias color marrón evidenciadas por el medio ASG, de las cuales 109 cepas son identificadas bioquímicamente como $C$. neoformans y 3 cepas como $C$. gattii con el medio CGB (ver tabla 1 y figura 1). El índice Prz para proteinasas y fosolipasas de las cepas aisladas, se exponen en la tabla 2.
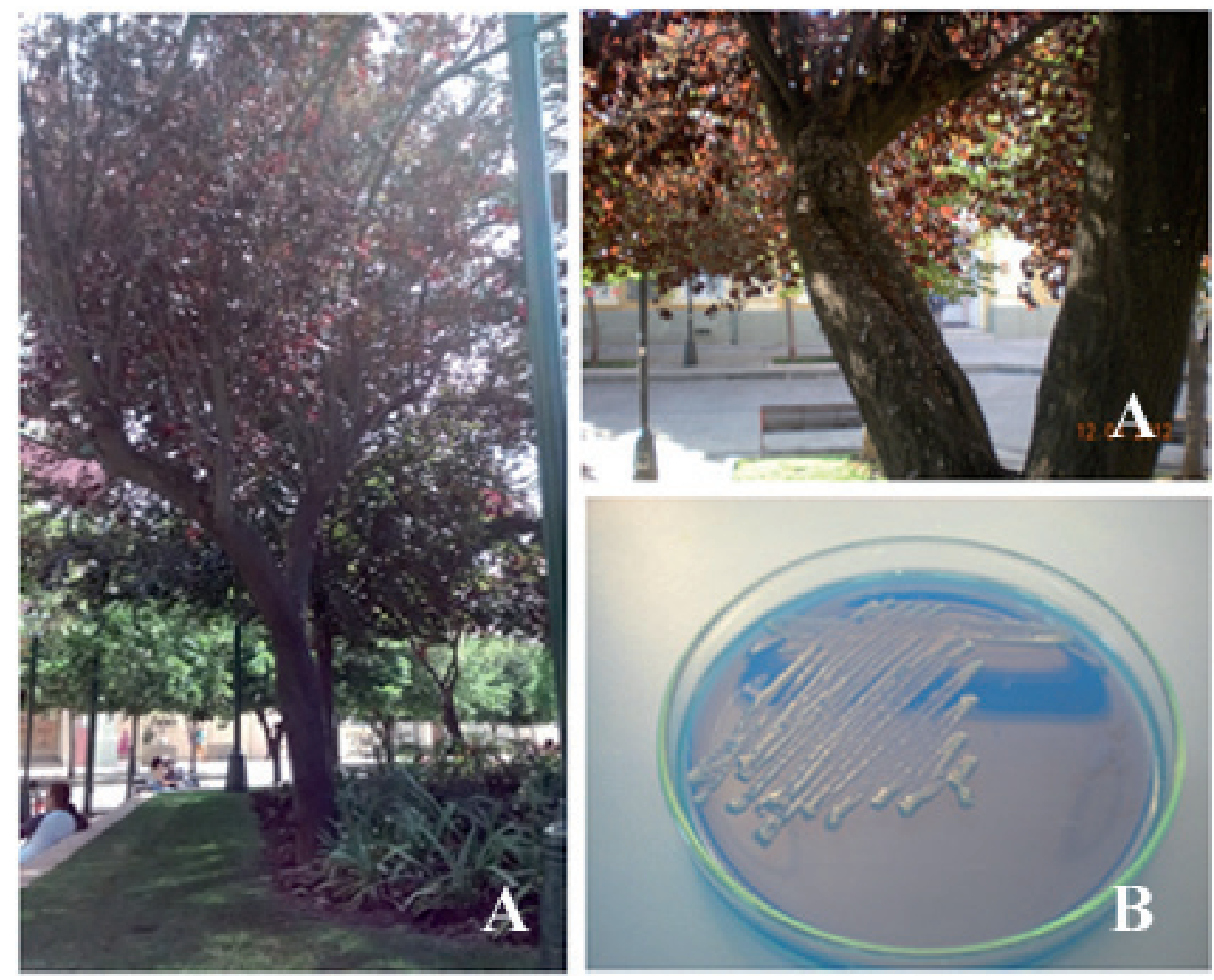

Figura 1. (B) Muestra presuntiva de $C$. gattii $(\mathrm{CGB}+$ ) aislada desde la plaza de Armas de Rancagua, se observa la mucosidad característica de las colonias, por la presencia de la cápsula. (A) Prunus cerasifera atropurpurea (ciruelo morado) desde donde se extrajo la cepa. 
Tabla 1. Localidades, número de muestras y cepas de $C$. neoformans y $C$. gattii aisladas desde oquedades de distintas especies arbóreas.

\begin{tabular}{|c|c|c|c|c|}
\hline \multirow[t]{2}{*}{ Región } & \multirow[t]{2}{*}{ Sector/ Localidad } & \multirow{2}{*}{$\begin{array}{c}\mathrm{N}^{\circ} \text { de } \\
\text { muestras }\end{array}$} & \multicolumn{2}{|c|}{ Identificación bioquímica } \\
\hline & & & C. neoformans & C. gattii \\
\hline \multirow{9}{*}{$\begin{array}{l}\text { Región } \\
\text { de } \\
\text { O’Higgins }\end{array}$} & Coya/Machalí & 16 & 0 & 0 \\
\hline & Tierras blancas/ Machalí & 4 & 0 & 0 \\
\hline & Cerro San Juan/ Machalí & 10 & 0 & 2 \\
\hline & Plaza de Armas/ Machalí & 2 & 32 & 0 \\
\hline & Plaza de Armas/ Mostazal & 2 & 0 & 0 \\
\hline & Plaza de Armas/Graneros & 6 & 4 & 0 \\
\hline & Estadio El Teniente/ Rancagua & 16 & 4 & 0 \\
\hline & Plaza de Armas/ Rancagua & 20 & 3 & 1 \\
\hline & Alameda/ Rancagua & 40 & 59 & 0 \\
\hline Región del & Plaza E.F.E./ Talca & 8 & 4 & 0 \\
\hline \multirow[t]{3}{*}{ Maule } & Universidad de Talca/ Talca & 30 & 1 & 0 \\
\hline & Alameda/ Talca & 32 & 0 & 0 \\
\hline & Plaza de Armas/ Talca & 14 & 2 & 0 \\
\hline \multicolumn{2}{|r|}{ Total } & 200 & 109 & 3 \\
\hline
\end{tabular}

Tabla 2. Rango de actividad enzimática de proteinasas y fosfolipasas, de cepas aisladas desde oquedades de especies arbóreas.

\begin{tabular}{|l|cc|cc|}
\hline \multirow{2}{*}{$\begin{array}{l}\text { Rango de } \\
\text { actividad } \\
\text { (Prz) }\end{array}$} & \multicolumn{4}{c|}{ Proteinasas } \\
\cline { 2 - 5 } & C. neoformans & C. gattii & C. neoformans & C. gattii \\
\hline Alta $(\leq 0,69)$ & $96(88,1)$ & $3(100)$ & $54(49,5)$ & $1(33,3)$ \\
Media $(0,7-0,89)$ & $9(8,3)$ & $0(0)$ & $39(37,8)$ & $0(0)$ \\
Baja $(0,9-0,99)$ & $2(1,8)$ & $0(0)$ & $3(2,8)$ & $1(33,3)$ \\
Nula $(1)$ & $2(1,8)$ & $0(0)$ & $13(11,9)$ & $1(33,3)$ \\
& $109(100)$ & $3(100)$ & $109(100)$ & $3(100)$ \\
\hline
\end{tabular}

\section{DISCUSIÓN}

La criptococosis se describe como una micosis sistémica causada por pequeños propágulos inhalables de C. neoformans en pacientes inmunocomprometidos y $C$. gattii en inmunocompetentes. $C$. neoformans es una levadura encapsulada que ha sido aislada, principalmente, desde las excretas de las aves, distribuido a nivel mundial. Además, se ha encontrado como saprofito en frutas, leche de animales, madera, suelo, pasto, establos y tubérculos como las papas y las zanahorias (9). $C$. 
Aislamiento presuntivo y caracterización de Cryptoccoccus neoformans y Criptococcus gatti - V. Toro et al

gattii, aislado por primera vez en 1990 por Ellis y Pfeiffer en Australia desde la corteza, hojas y restos vegetales depositados debajo de Eucalyptus camaldulensis en floración (1), se ha esparcido, presumiblemente, junto a semillas y tallos jóvenes de especies de eucaliptus a otras partes del mundo, de preferencia con características climáticas tropicales y subtropicales. Springer \& Chaturvedi (34), informan el aumento de reportes de casos clínicos de C. gattii en animales nativos y domésticos en regiones templadas. Hasta el año 2010, se reconocen unas 54 especies de árboles nativos, desde donde se ha aislado $C$. gattii, ubicadas en regiones tropicales, subtropicales y templadas.

En el presente estudio, 109 cepas aisladas desde las oquedades de distintas especies arbóreas fueron identificadas bioquímicamente como $C$. neoformans. Y desde el cerro San Juan en Machalí se logró recuperar dos cepas CGB positiva desde una especie de Eucalyptus sp. , y una cepa en la plaza de Armas de Rancagua desde una especie de ciruelo morado (Prunus cerasifera artropurpurea). Una de las características comunes de estas zonas de muestreo, es la gran afluencia de público, por ser lugares de esparcimiento y recreación, razón por la cual cobra importancia el estudio ecológico de esta levadura. Las zonas mencionadas, presentan un clima templado cálido con lluvias invernales y estación seca prolongada y una amplitud térmica anual que supera $\operatorname{los} 13^{\circ} \mathrm{C}(3)$. Ordoñez y col.(28) en la ciudad de Cúcuta Colombia, trató de establecer la fuente ambiental de $C$. gattii serotipo $\mathrm{B}$, debido a la alta frecuencia de casos clínicos en esta ciudad. Recolectaron 1.736 muestras a partir de detritos de Eucalyptus, Moquilia (oiti), Delonix (acacia) y Terminalia (almendros) y material vegetal alrededor de estos, por un período de 11 meses, recuperando 40 aislamientos de $C$. neoformans var. neoformans y una tasa de recuperación de aislamientos del complejo C. neoformans/C. gattii del 2,3 $310-2 \%$. En este estudio no se identifica el serotipo de las cepas de $C$. gattii, pero coincide con la baja proporción de cepas de $C$. gattii encontradas en el ambiente, comparativamente con las cepas de C. neoformans.

C. neoformans se aísla frecuentemente desde el ambiente, sin depender de la especie arbórea, y $C$. gattii ha ampliado su nicho ecológico, siendo encontrado en diversas especies de árboles, como lo fue en este estudio, logrando una cepa aislada desde Prunus cerasifera artropurpurea (ciruelo morado). Torres en el 2010 (37), realiza un muestreo exploratorio en la zona de Cúcuta (Colombia) aísla C. gattii serotipo B, no determinando con exactitud el tipo de árbol del cual extrajo las muestras, pero teniendo en cuenta que en la zona de muestreo se caracteriza por presentar arboles de Ficus y Terminalia cattapa (Almendro). Además, en las mismas zonas logró recuperar aislamientos de $C$. neoformans serotipo A (var. grubii) y un aislamiento de $C$. gattii serotipo C. La distribución de C. gattii reportada hasta el 2010 por Springer \& Chaturvedi (34) es tan diversa, incluye a países como Argentina, Austria , Canadá, China , Congo, India, Italia, Japón, Corea del Sur, Holanda, España, Sudáfrica, Reino Unido, Estados Unidos, y Democrática República del Congo, ampliándose cada año. Sin embargo, no se han reportado aislamientos clínicos o ambientales de $C$. gattii en Chile.

Tomando en cuenta el clima templado y el inicio del otoño, en que se realizó el muestreo, favoreció la posibilidad de encontrar a $C$. neoformans y $C$. gattii, de acuerdo a un estudio retrospectivo de la influencia del clima en la proliferación ambiental de las cepas, en las épocas del año con alta precipitación y humedad, pocas horas de sol, bajas temperaturas y poca fluctuación de temperatura favorece la ocurrencia de $C$. gattii serotipo B asociada a diversos árboles. C. neoformans var. grubii serotipo A y $C$. gattii serotipo $\mathrm{C}$, se favorecen en épocas secas del año, con valores bajos de humedad, altas temperaturas, poca precipitación, periodos largos de sol y cambios extremos de temperatura (19). Es importante que en estudios posteriores, se identifiquen los serotipos y se pueda muestrear en distintas épocas del año.

La mayoría de los estudios de C. neoformans en nuestro país están asociados a casos clínicos, prin- 
cipalmente en pacientes con algún grado de compromiso inmunológico $(20,18)$. La producción de proteinasas ha implicado la patogénesis de varios hongos y bacterias. Las proteinasas contribuyen a la destrucción de los tejidos del huésped, digiriendo proteínas inmunológicamente importantes, como los anticuerpos y el complemento. Se reconoce la presencia de proteinasas extracelulares en C. neoformans como medio de crecimiento hidrolizando las proteínas en agar (10). De las cepas aisladas, el 88,1\% (índice de producción $\leq 0,69$ ) de C. neoformans y el $100 \%$ de C. gattii, presentó un alto índice de actividad de proteinasas extracelulares. Sánchez y col. (35) midió proteinasas extracelulares a 54 aislamientos clínicos de C. neoformans de pacientes VIH positivos y 19 cepas de C. gattii, uno VIH positivos y el resto de pacientes VIH negativos. El 37,1\% de las cepas C. neoformans presentó una alta actividad de enzimática, el $48,6 \%$ actividad media y un $14,3 \%$ con actividad baja. Para las cepas de C. gattii el $21 \%$ presentó actividad enzimática alta, el $47 \%$ actividad media y $32 \%$ actividad baja. En contraste, en este estudio, las 3 cepas de $C$. gattii. En otro estudio que compara actividad de enzimas entre cepas de $C$. gatti Colombianas y provenientes de Vancouver, no se encontró actividad proteolítica en las cepas de Colombia y el grupo de Vancouver presentó actividad baja (índice Prz de 0,77) (37). Las diferencias entre las actividades enzimáticas de los aislados, puede deberse al tipo y cantidad de proteínas producidas por cada cepa, lo cual puede influenciar al desarrollo de la criptocococis (36).

La capacidad de ciertos hongos patógenos de producir y excretar enzimas fosfolipásicas es considerada como un potencial factor de virulencia y patogenicidad, permitiendo a los microorganismos la capacidad de diseminarse (15). En C. gattii, la acción de esta enzima desestabiliza las membranas, provoca lisis celular y liberación de segundos mensajeros lipídicos, permitiendo la invasión tisular (37). En este estudio el 49,5\% (índice de producción $\leq 0,69)$ de las cepas, presentó un alto índice de actividad de fosfolipasas, esta cifra difiere a lo obtenido por Vidotto et al. (40) el cual obtiene un índice alto en un $78 \%$ de las cepas testeadas, aisladas desde pacientes con SIDA. Además, Echeverría y col. (15) probando medios de ASD yema de huevo con cepas de C. neoformans, observa que el $85 \%$ de las cepas son capaces de producir y liberar enzimas con capacidad de fofolipasas. Estos resultados, podrían demostrar la baja virulencia de las cepas ambientales. Las tres cepas $C$. gattii aisladas presentaron una actividad de fosfolipasa muy distinta entre ellas. Una cepa aisladas desde Eucalyptus sp. del cerro San Juan en Machalí, presentó una baja actividad (índice de 0,89 ) y la única cepa aislada desde un ciruelo morado (Prunus cerasifera artropurpurea ) de la plaza de Armas de Rancagua, mostró un alto índice de actividad de fosfolipasas (índice de 0,53). Estos resultados coinciden con un estudio realizado por Huérfano y col. (21) quienes aislaron desde detritos de almendros cepas de $C$. neoformans variedad grubii serotipo A y C. gattii serotipo C, de los 31 aislamientos de C. gattii evaluados el $58 \%$ de los aislamientos no presentaban actividad y un $42 \%$ presentaba actividad muy baja, además, en este estudio no encontraron relación directa entre la producción de fosfolipasas y la virulencia de las cepas. Finalmente, es necesario realizar nuevos muestreos en otras épocas del año y completar los estudios con datos genéticos, considerando los importantes cambios climáticos que podrían favorecer la proliferación de esta levadura y las posibilidades de incidencia de criptococosis en la población.

\section{REFERENCIAS}

1. Agüero B., Garza D., Flores V. y Cervantes R. (1999). Aislamiento y caracterización de Cryptococcus neoformans var. gattii a partir de muestras de Eucalyptus camaldulensis en la ciudad de México. Rev Iberoam Micol. 16:40
2. Alvarado E. (2008). Estudio de los Factores de Patogenicidad de Cryptococcus gattii. Tesis Doctoral. Universidad Autónoma de Barcelona. Departamento de genética y Microbiología. 
Aislamiento presuntivo y caracterización de Cryptoccoccus neoformans y Criptococcus gatti - V. Toro et al

3. Alvarado G. y Moya C. (2007). División político-administrativa y censal, 2007. Instituto Nacional de Estadística INE Chile.

4. Arredondo C. (2006). Cryptococcus neoformans en heces de palomas recolectadas en lugares públicos de la ciudad de Santiago, Chile. Trabajo de investigación para optar al título de Médico Veterinario. Universidad Iberoamericana de Ciencias y Tecnología. Santiago de Chile.

5. Boekhout T, Van Belkum A, Leenders A, Verbrugh H, Mukamurangwa $P$, Swinne D. and Scheffers A. (1997). Molecular Typing of Cryptococcus neoformans: Taxonomic and Epidemiological Aspects. Int J Syst Bacteriol. 47(2):432-442.

6. Barnett J. (2010). A history of research on yeasts: medical yeasts part 2, Cryptococcus neoformans. Yeast. 27: 875-904.

7. Baró M. (2002). Epidemiología de la Criptococosis en España. Caracterización de los aislados de Cryptococcus neoformans. Tesis para optar al grado de Doctor en Ciencias Biológicas. Universitat Autónoma de Barcelona.

8. Bovers M, Hagen F. and Boekhout T. (2008). Diversit of the Cryptococcus neoformans-Cryptococcus gattii species complex. Rev Iberoam Micol. 25: S4-S12.

9. Curo M, Salinas M. y Casquero J. (2005) Cryptococcus neoformans en excretas de palomas, suelo y aire de los palomares del perímetro urbano del Ica, 2002. Rev. Perú Med Exp salud pública. 22 (4).

10. Chen L, Blank E. and Casadevalli A. (1996). Extracellular Proteinase Activity of Cryptococcus neoformans. Clinical and diagnostic laboratory inmunology. 3 ; 5:570-574

11. Datta K, Bartlett K, Baer R, Hoang L, Byrnes E, Galanis E, Heitman J, Leslie M, MacDougall L, Magill S, Morshed M and Marr K.(2009).
Spread of Cryptococcus gattii into Pacific Northwest Region of the United States. Emerging Infectious Diseases 15(8).

12. Dromer F, Guého E, Ronin O, Dupont B.(1993) Serotyping of Cryptococcus neoformans by using a monoclonal antibody specific for capsular polysaccharide. J. Clin. Microbiol. 31(2):359363.

13. Ellis D. and Pfeiffer T. (1990). Natural Habitat of Cryptococcus neoformans var. gattii. J Clin Microbiol. 28(7): 1642-1644.

14. Escandon P, Quintero E, Granados D, Huérfano S, Ruiz A. y Castañeda E. (2005). Aislamineto de Cryptococcus gatti serotipo B desde detritus de Eucalyptus spp. en Colombia. Biomédica 25 (3): 390-397.

15. Echeverría A, Durante A, Arechavala A. y Negroni R. (2002). Estudio comparativo de dos medios de cultivo para la detección de la actividad fosfolipasa en cepas de Candida albicans y Cryptococcus neoformans. Rev Iberoam Micol 19: 95-98.

16. Evans E. (1950). The antigenic composition of Cryptococcus neoformans. I. A serologic classification by means of the capsular and agglutination reactions. J. Immunol 64:423-430.

17. Franzot S, Salkin I and Casadevall A. (1999). Cryptococcus neoformans var. grubii: Separate Varietal Status for Cryptococcus neoformans Serotype A Isolates. J Clin Microbiol 37(3): 838-840.

18. Gil G, Foster C, Neira O, Palma S, Basualdo J, Heredia C, Fernández A, Juliet $\mathbf{C}$ y Tapia J.(2006). Artritis por Cryptococcus neoformans en un adulto mayor: Presentación de un caso y revisión. Rev Chil Infect 23 (4): 330-335.

19. Granados D. and Castañeda E. (2006). Influence of climatic conditions on the isolation of members of the Cryptococcus neoformans species 
Aislamiento presuntivo y caracterización de Cryptoccoccus neoformans y Criptococcus gatti - V. Toro et al

complex from trees in Colombia from 1992-2004. Fems Yeast Res. 6: 636-644.

20. Hirsch T, Hannig S y Yañez L. (2000) Meningoencefalitis por cryptococcus. Reporte de un caso clínico y revisión de la literatura. Rev chil de pediatr.71(4).

21 .Huérfano S, Cepero $M$ y Castañeda E. (2003). Caracterización fenotípica de aislamientos ambientales de Cryptococcus neoformans. Biomédica. 23: 328-40.

22. Kidd S, Chow Y, Mak S, Bach P, Chen H, Hingston A, Kronstad J and Bartlett K. (2007). Characterization of Environmental Sources of the Human and Animal Pathogen Cryptococcus gattii in British Columbia, Canada, and the Pacific Northwest of the United States. Appl Environ Microbiol. 73(5): 1433-1443.

23. Kwon-Chung KJ, Polacheck I and Bennett J. (1982). Improved Diagnostic Medium for Separation of Cryptococcus neoformans var. neoformans (Serotypes A and D) and Cryptococcus neoformans var. gattii (Serotypes B and C). J Clin Microbiol. 15(3): 535-537.

24 .Kwon-Chung KJ, Boekhout T, Fell JW \& Diaz M. (2002). Proposal to conserve the name Cryptococcus gattii against $\mathrm{C}$. hondurianus and $\mathrm{C}$. bacillisporus (Basidiomycota, Hymenomycetes, Tremellomycetidae). Taxon 51: 804-806.

25. Littman M. (1959). Cryptococcosis (Torulosis): Current concepts and therapy. Am. J. Med. 27: 976-998.

26. Nayak J, Brahmbahtt M, Savalia C, Pal M y Bhanderi.(2010). Cryptococcosis: A Garded Mycosis Threat. Research Journal of Veterinary Sciences. 3: 101-112.

27. Okabayashi K, Kano R, Nakamura Y, Wata- nabe S, Hasegawa A. (2006). Capsule-associated genes of serotypes of Cryptococcus neoformans, especially serotype AD. Med Mycol. 44(2):12732.

28. Ordoñez N, Rodriguez M, et al. (1997). Search for the habitat of Cryptococcus neoformans var. gattii in a northeast city in Colombia. Preliminary data. Abstract's book of the 13th congress of the International Society for Human and Animal Mycology. Salsomaggiore Terme, Parma, Italia.

29. Panizo M, Revianka V, Flores Y, Montes W y González G.(2005). Actividad de fosfolipasas y protesasas en aislados clínicos de Candida spp. Rev. Soc. Ven. Microbiol. 25(2):64-71.

30. Price M, Wilkinson I, Gentry L.(1982). Plate method for detection of phospholipase activity in Candida albicans. Sabouraudia 20: 7-14.

31. Pumarola A, Rodríguez A, García J y Piedrola G. (1991). Microbiología y Parasitología Médica. $2^{\circ}$ Edición. Editorial Salvat Editores. Barcelona. 786 .

32. Ruma-Haynes P, Brownlee A and Sorrell T.(2000). A rapid method for detecting extracellular proteinase activity in Cryptococcus neoformans and survey of 63 isolates. J. Med. Microbiol. 49: 733-737.

33. Springer D and Chaturvedi V. (2010). Projecting Global Occurrence of Cryptococcus gattii. Emerg Infect Diseases. 16(1).

34. Steenbergen J and Casadevall A.(2003). The origin and maintenance of virulence for the human pathogenic fungus Cryptococcus neoformans. Microbes Infect. 5: 667-75.

35. SánchezA, Escandón Py Castañeda E.(2008). Determinación in vitro de la actividad de los factores asociados con la virulencia de aislamientos 
clínicos del complejo Cryptococcus neoformans. Rev Iberoam Micol. 25(3): 145-149.

36. Torres G. (2010). Estudio de las características genotípicas y fenotípicas entre aislamientos colombianos de Cryptococcus gattii serotipo B-patrón VGII, procedentes de Cúcuta y aislamientos responsables de la epidemia e Vancouver Canadá. Maestría en Microbiología. Facultad de Ciencias. Universidad Nacional de Colombia.

37. Torres J, Alvarado E, Gutiérrez R.(2008). Diferencias en la actividad de la enzima ureasa en- tre Cryptococcus neoformans y Cryptococcus gattii. Rev Iberoam Micol. 25: 27-31.

38. Urán M. \& Cano L.(2008). Melanina: implicaciones en la patogénesis de algunas enfermedades y su capacidad de evadir la respuesta inmune del hospedero. Asociación Colombiana de Infectología. 12-2.

39.Vidotto V, Sinicco A Di Fraia D, Cardaropoli S, Aoki S y Ito-Kuwa S. (1996). Phospholipase activity in Cryptococcus neoformans. Mycopathologia 136(3): 119-123. 